\title{
MODEL ATMOSPHERES FOR COOLING NEUTRON STARS
}

\author{
Roger W. Romani and Roger D. Blandford \\ California Institute of Technology \\ Pasadena, California 91125 \\ and Lars Hernquist \\ University of California, Berkeley \\ Berkeley, California 94720
}

Abstract. The failure of Einstein X-ray observations to detect
central neutron stars in most young supernova remnants
(Helfand and Becker 1984) has provided interesting
constraints on cooling theories (cf. review by Tsuruta 1985).
The comparison of the measured fluxes with the predicted
effective temperatures is sensitive to the nature of the
emitted spectrum, commonly assumed to be blackbody. The
presence of a substantial absorbing atmosphere can, however, produce significant departures. We have calculated model atmospheres for unmagnetized neutron stars with effective temperatures $10^{5} \mathrm{~K} \leqq T_{\text {eff }} \leqq 10^{6.5} \mathrm{~K}$ using Los Alamos opacities and equations of state (Romani 1986). We consider a range of surface compositions, since the accretion of $\sim 10^{-19} M_{\odot}$ will cover the surface to the X-ray photosphere and subsequent settling in the strong gravitational field can severely deplete the heavy species. In a low Z atmosphere (eg. $\mathrm{He}$ ) the measured X-ray flux will substantially exceed the blackbody value--the Einstein limits on $T_{\text {eff }}$ are correspondingly lowered (eg. by $\sim 1.6$ for SN 1006 with a helium surface). For high $\mathrm{Z}$ atmospheres, the flux is close to the black body value, but prominent absorption edges are present. Recent calculations of the electron heat transport in magnetized neutron star envelopes (Hernquist 1984, 1985) have shown that, contrary to earlier estimates, magnetic fields will have a small effect on the heat flux ( $\leqslant 3$ for parallel field geometries and $\sim 1$ for tangled fields). Extension of the atmosphere computations to the magnetic case is important for comparison with X-ray observations of known pulsars.

\section{References}

Helfand, D. J. and Becker, R. 1984. Nature, 307, 215.

Hernquist, L. 1984. Ap. J. Suppl., 56, 325.

Hernquist, L. 1985. Mon. Not. R. Ast. Soc., 213, 313.

Romani, R. W. 1986. Ap. J., submitted.

Tsuruta, S. 1985. Max-Planck Institut Für Astrophysik preprint. 